\title{
Molecular markers of tuberculosis and their clinical relevance: a systematic review and meta-analysis
}

\author{
Feifan $\mathrm{Xu}^{1,2 \#}$, Ming $\mathrm{Ni}^{2,3 \#}$, Shengyan $\mathrm{Qu}^{1}$, Yinong Duan ${ }^{2}$, Haiyun Zhang ${ }^{1}$, Zhihua Qin ${ }^{4}$ \\ ${ }^{1}$ Department of Clinical Laboratory, Affiliated Nantong Hospital of Shanghai University, Nantong, China; ${ }^{2}$ Department of Pathogen Biology, School \\ of Medicine, Nantong University, Nantong, China; ${ }^{3}$ Department of Clinical Laboratory, Disease Control and Prevention Center of Rugao, Nantong, \\ China; ${ }^{4}$ Department of Tuberculosis, Affiliated Nantong Hospital of Shanghai University, Nantong, China \\ Contributions: (I) Conception and design: F Xu; (II) Administrative support: M Ni, S Qu; (III) Provision of study materials or patients: Y Duan, H \\ Zhang; (IV) Collection and assembly of data: Z Qin; (V) Data analysis and interpretation: H Zhang, Z Qin; (VI) Manuscript writing: All authors; (VII) \\ Final approval of manuscript: All authors. \\ \#These authors contributed equally to this work and should be considered as co-first authors. \\ Correspondence to: Haiyun Zhang. Department of Clinical Laboratory, Affiliated Nantong Hospital of Shanghai University, Nantong 226011, China. \\ Email: zhy34@163.com; Zhihua Qin. Department of Tuberculosis, Affiliated Nantong Hospital of Shanghai University, Nantong 226011, China. \\ Email: ntlyxff@163.com.
}

Background Since research on disease biomarkers of tuberculosis (TB) and latent tuberculosis infection (LTBI) provides hope for simple point-of-care testing, we aim to summarize and analyze the evidence for the clinical relevance of IFN- $\gamma$-inducible protein 10 (IP-10) and IFN- $\gamma /$ interleukin 2 (IL-2) as diagnostic biomarkers for TB.

Methods: The search terms tuberculosis, tuberculous pleurisy, pulmonary tuberculosis, latent tuberculosis infection, biomarkers, markers, IFN- $\gamma$-inducible protein 10, IP-10, interleukin 2, and IL-2 were used to search the PubMed, Cochrane Central Register of Controlled Trials, EMBASE, Web of Science, China National Knowledge Infrastructure (CNKI), Wanfang, and Weipu databases. The retrieval time was from the establishment of the database to September 2021. The Cochrane risk of bias tool was used to evaluate the quality of the included studies, and the meta-analysis was performed using RevMan 5.20.

Results: A total of 9 articles were included for meta-analysis. The quality assessment showed that the overall quality of the included articles was met the requirements. The results showed that the overall sensitivity and specificity of IP-10 were 0.77 (95\% CI, 0.71-0.82) and 0.84 (95\% CI, 0.80-0.88), respectively. The overall sensitivity and specificity of IL-2 were 0.82 (95\% CI, 0.74-0.89) and 0.95 (95\% CI, 0.88-0.98), respectively. The areas under the curves (AUCs) of the IP-10 and IL-2 summary receiver operating characteristic (SROC) curves were 0.8592 and 0.9666 , respectively.

Discussion: The results of this systematic review and meta-analysis showed that IP-10 and IL-2 as biomarkers have good clinical relevance to TB and can be used for the clinical screening of high-risk TB populations. However, a prospective cohort study across multiple regions using a large sample size should also be conducted.

Keywords: Biomarkers; IFN- $\gamma$-inducible protein 10 (IP-10); interleukin 2 (IL-2); tuberculosis; mycobacterium tuberculosis

Submitted Nov 19, 2021. Accepted for publication Jan 30, 2022.

doi: 10.21037/apm-21-3739

View this article at: https://dx.doi.org/10.21037/apm-21-3739 


\section{Introduction}

Tuberculosis (TB) kills 4,000 people every day all over the world $(1,2)$. The World Health Organization $(\mathrm{WHO})$ and researchers in this field are still looking for solutions that can control and prevent TB. Because TB occurs more often in young adults, this disease has caused significant economic and medical problems. In 2014, the WHO approved a new strategy with the goal of ending the global TB epidemic and eliminating TB by 2035 (3-5). This requires a reduction in the incidence of TB per 100,000 people to less than 10 in order to achieve this goal $(6,7)$. Therefore, the discovery, development, and rapid implementation of new diagnostic tools have been identified as an important part of the WHO's strategy to end TB $(4,7,8)$.

The 2 major strategies for TB control are the early diagnosis and treatment of patients with active TB and preventive treatment of latent tuberculosis infection (LTBI) (9-11). One of the first challenges for TB control is accurate diagnosis (12). The gold standard for tuberculosis diagnosis is mainly to find Mycobacterium tuberculosis in sputum or during culture, but this is not easy to implement. Until now, the tuberculin skin test (TST) has been the traditional method used to detect LTBI (13). Although it is fast and cheap, TST has low specificity (14). Furthermore, the technology has some limitations, such as low sensitivity in immunodeficiency patients, the need for return visits, variation between readers, and compatibility with bacille calmette-guerin (BCG) vaccines and non-TB strains. Cross-reaction with NTM results in false positive results $(9,15,16)$. In addition, studies have shown that about $10 \%$ of LTBI patients may develop active TB infection in their lifetime $(9,13,15)$, and this proportion will increase in immunocompromised patients, such as HIV-positive individuals and organ transplant patients with cell-mediated immune damage (17).

Recent studies have shown that the combined use of alternative cytokines and IFN- $\gamma$ may increase the sensitivity of interferon gamma (IFN- $\gamma$ ) release assays (IGRAs) (18). IFN- $\gamma$-inducible protein 10 (IP-10) has been identified as a sensitive marker for detecting previous $M$. tuberculosis infection in children and adults (18). Although IGRAs cannot distinguish between TB and LTBI in children $(19,20)$, recent studies have found that a modified IGRA based on the simultaneous analysis of a series of different markers may be more effective. These studies have identified a variety of cytokine combinations, such as IFN- $\gamma /$ interleukin (IL)-2, IL-17/IL-10, IL-15/monocyte chemotactic protein (MCP1), tumor necrosis factor alpha
(TNF- $\alpha$ )/IL-2 (9,21-24), and even more complex T cell effector molecular patterns (25), which are used as possible biomarkers to distinguish between TB and LTBI in adults and children.

Therefore, the purpose of this systematic review is to evaluate the potential value of IP-10 as a marker for distinguishing TB patients and healthy people, and to evaluate the value of IL-2 as a marker for distinguishing LTBI patients and healthy people, with a view to determine the clinical value of the combined use of these biomarkers for the diagnosis of TB and LTBI. We present the following article in accordance with the PRISMA-DTA reporting checklist (available at https://apm.amegroups.com/article/ view/10.21037/apm-21-3739/rc).

\section{Methods}

\section{Search strategy}

According to the aim of the study, we analyzed the clinical correlation between IP-10 and IL-2 biomarkers and TB. Literature searches were conducted using the English biomedical databases PubMed, Cochrane Central Register of Controlled Trials, EMBASE, and Web of Science, and the major Chinese biomedical databases CNKI, Wanfang, and Weipu. The following search terms were used to search the databases: tuberculosis, tuberculous pleurisy, pulmonary tuberculosis, latent tuberculosis infection, biomarkers, markers, IFN- $\gamma$-inducible protein 10, IP-10, interleukin 2, and IL-2. The language was limited to Chinese and English. The retrieval time was from the establishment of the database to September 2021.

\section{Inclusion and exclusion criteria}

Studies were included based on the following criteria: studies on humans or human biological samples; studies using IP-10, IL-2 or IP-10, and IL-2 to diagnose or screen TB; studies published in Chinese and English. Studies were excluded based on the following criteria: elderly population; patients had other serious diseases that affect the body's immunity; index tests that required imaging techniques or bacterial culture detection.

\section{Literature screening and data extraction}

Two reviewers independently evaluated the titles and abstracts of the articles to determine whether they were eligible to be selected for the study. If either reviewer 
believed that the article met the criteria, the full texts would be reviewed. If there was a disagreement about the eligibility of the article, the 2 reviewers would discuss whether the eligibility of the article could be agreed upon. If the discussion failed to reach a consensus, a third reviewer would be used for the decision. If additional information on the article was required, the reviewers contacted the author of the article. The data was also independently extracted by the 2 reviewers in accordance with pre-established data tables. Data including author name, country, publication time, journal name, and biomarker were extracted.

\section{Quality assessment}

The included articles were evaluated for quality, and the scope of the evaluation included patient selection, index testing, reference standards, flow, and time. Each indicator was evaluated based on the risk of bias, and the first 3 indicators were evaluated based on applicability issues. Each methodological quality was rated as "low risk", "high risk", or "unclear". The Quality Assessment of Diagnostic Accuracy Studies-2 (QUADAS-2) recommended by the Cochrane Collaboration was used to assess the methodological quality of the research. Two reviewers carried out the above process separately, and if there was any difference, it would be discussed and resolved by the 2 reviewers.

\section{Statistical analysis}

The sensitivity, specificity, positive likelihood ratio (PLR), negative likelihood ratio (NLR), diagnostic odds ratio (DOR), and area under the receiver operating characteristic (ROC) curves (AUC) with their 95\% confidence intervals (CIs) for each individual study of IP-10 and IL-2 were calculated for TB, according to the main outcome indicators extracted from the enrolled studies. The hierarchical logistic regression model was used to calculate general estimates of the sensitivity and specificity of the enrolled study, including the hierarchical summary receiver operating characteristic (SROC) model. SROC curves with 95\% CIs and prediction regions were used to map the results for sensitivity and specificity. The Chi-square test and Higgins $\mathrm{I}^{2}$ test were used to assess the heterogeneity of the included studies. PLR, NLR, and DOR were calculated by the bivariate generalized linear mixed model and the random effects model, and the sensitivity analysis was performed according to the Cochrane systematic review method. All analyses were performed by Stata 15.0 (StataCorp LLC, College Station, TX, USA) and
RevMan 5.20 software (Cochrane Collaboration), and a P value of $<0.05$ was considered statistically significant.

\section{Results}

\section{Search results and study characteristics}

The literature search yielded 549 records. After screening out 59 records, 490 records were obtained. Subsequently, 121 low-quality records were excluded again, and 369 records were retrieved to obtain 282 articles, including 204 English articles and 78 Chinese articles. After reading through the full texts and selecting articles according to the inclusion criteria, while excluding articles that did not meet the criteria and those with incomplete data, 9 articles were finally obtained. The specific screening process is shown in Figure 1.

Among the 9 articles used for analysis, 6 studies reported on the clinical relevance of IP-10 as a biomarker for TB. The subjects of the study were TB patients and healthy controls. Five studies reported the clinical relevance of IL-2 as a biomarker of LTBI. The subjects of the study were LTBI patients and healthy controls. The basic characteristics of the articles are shown in Table 1.

After rigorous quality and risk of bias assessments, it was found that for patient selection bias, 8 articles were described as low risk and 1 article was described as unclear risk. In terms of research index test bias, 4 articles were described as low risk while the risk in 5 articles was not described. All articles were considered low risk for reference standard bias. In terms of flow and time bias, 8 articles were described as low risk while the risk in 1 article was not described. Regarding the risk of applicability bias, patient selection bias, and reference standard bias, all articles were considered low risk. Index test bias was described as low risk in 8 articles, while the risk in 1 article was not described, as shown in Figure 2.

\section{Meta-analysis results}

\section{Overall analyses of IP-10}

The results of the meta-analysis showed that the sensitivity of the clinical correlation between IP-10 and TB was $\mathrm{I}^{2}=76.0 \%$, there is obvious heterogeneity. So, the combined analysis was performed using a random effects model, and the pooled sensitivity was 0.77 (95\% CI, 0.71-0.82) (Figure 3). The specificity $\mathrm{I}^{2}$ was $92.3 \%$, indicating obvious heterogeneity and the random effects model was used for 


\section{Identification of studies via databases and registers}

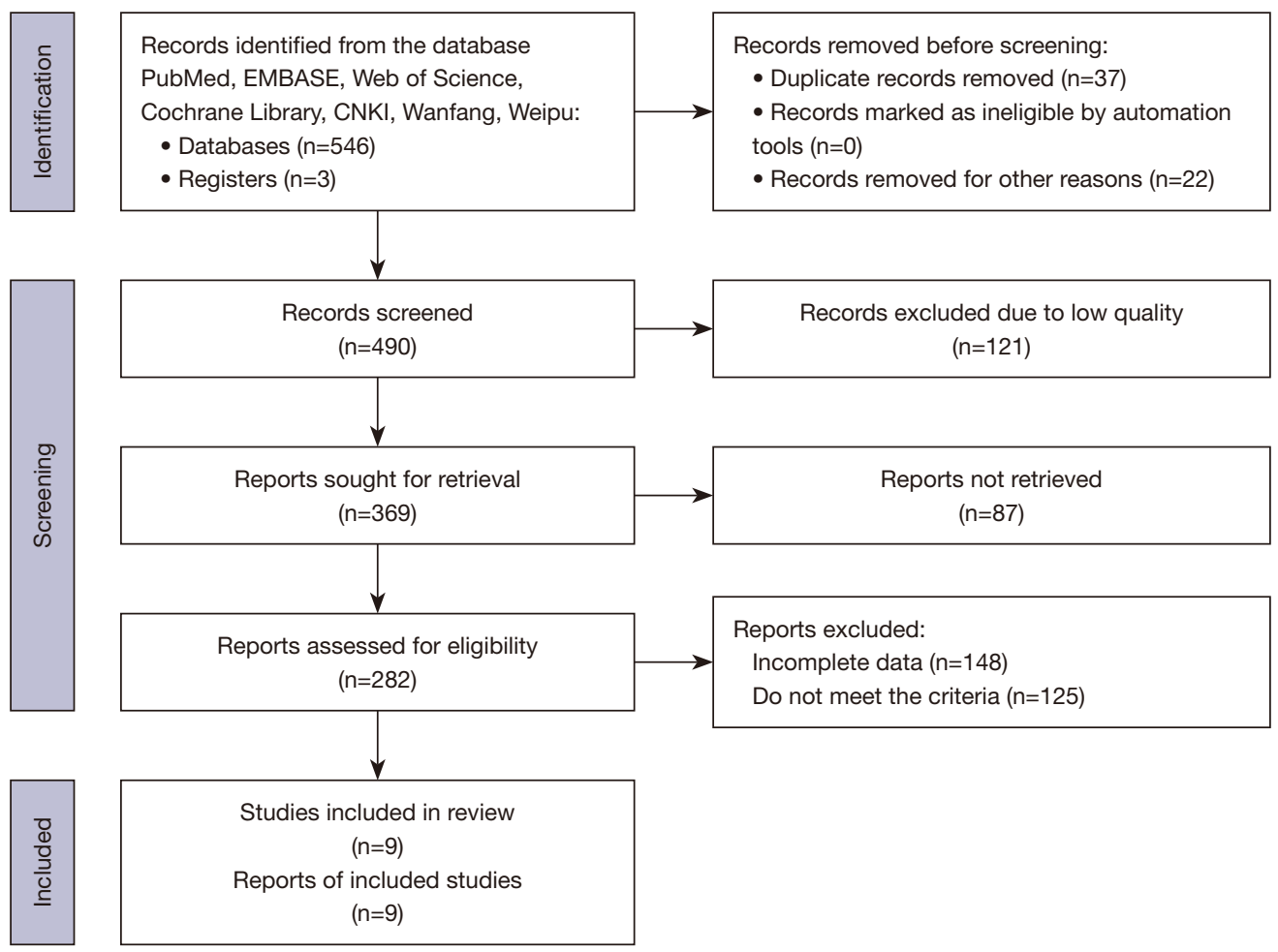

Figure 1 Flow diagram of the search, screening, and inclusion process.

Table 1 Basic characteristics of the studies

\begin{tabular}{|c|c|c|c|c|c|}
\hline Author & Country & Year & Journal & Biomarker & Population \\
\hline Luo et al. (27) & China & 2019 & $J$ Infect & IP-10 & $\mathrm{TB} / \mathrm{HC}$ \\
\hline Kamakia et al. (28) & Kenya & 2017 & Int $J$ Tuberc Lung Dis & IL-2 & LTBI/HC \\
\hline La Manna et al. (29) & Italy & 2018 & PLoS One & IP-10/IL-2 & TB/LTBI/HC \\
\hline Mamishi et al. (31) & Iran & 2019 & Mol Biol Rep & $\mathrm{IP}-10$ & $\mathrm{~TB} / \mathrm{HC}$ \\
\hline Nausch et al. (32) & Germany & 2017 & Int J Tuberc Lung Dis & IL-2 & LTBI/HC \\
\hline Nonghanphithak et al. (33) & Thailand & 2017 & Tuberculosis (Edinb) & $\mathrm{IP}-10$ & $\mathrm{~TB} / \mathrm{HC}$ \\
\hline Wang et al. (34) & China & 2018 & J Transl Med & IL-2 & LTBI/HC \\
\hline
\end{tabular}

TB, tuberculosis; LTBI, latent tuberculosis infection; HC, healthy controls; IP-10, IFN- $\gamma$-inducible protein 10; IL-2, interleukin 2.

combined analysis. The pooled specificity was 0.84 (95\% CI, 0.80-0.88) (Figure 4). The SROC curve showed higher accuracy (SAUC $=0.8592$ ) (Figure 5). The closer the AUC of the SROC curve is to 1.0 , the stronger the clinical correlation between IP-10 and TB.
The PLR is the probability of the correctly diagnosed disease and the incorrectly diagnosed disease in the diagnostic auxiliary examination, that is, the ratio of the positive rate of the diagnosed disease to the false positive rate. Therefore, in this study, the greater the PLR, the 


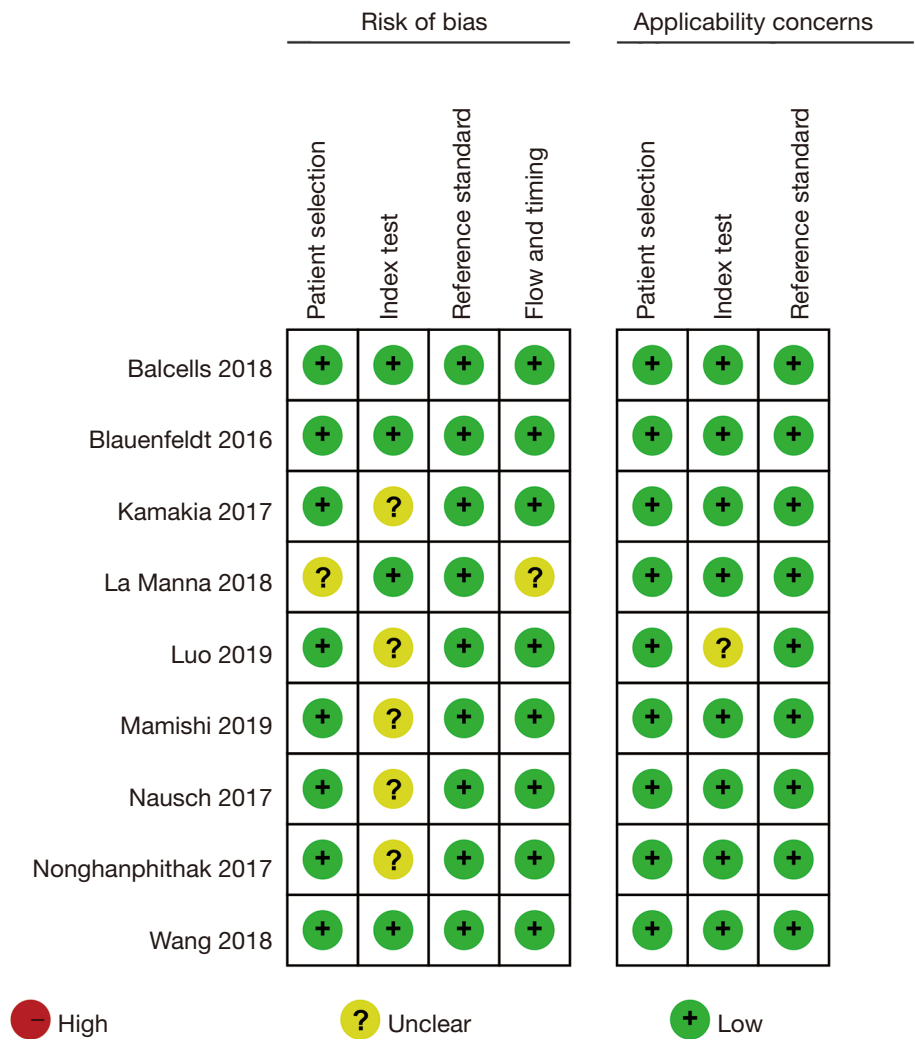

Figure 2 Literature quality evaluation details.
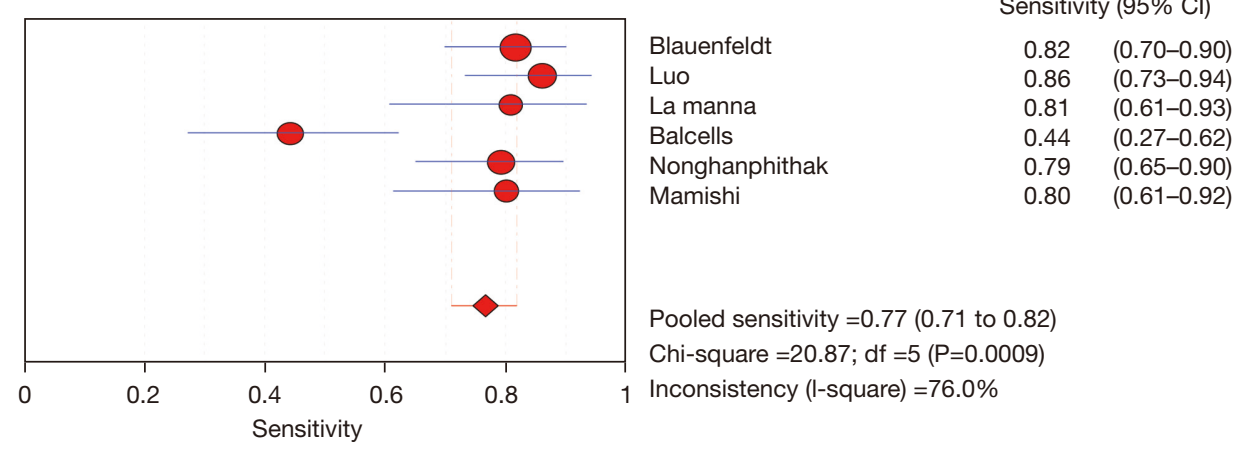

Figure 3 Forest plot of the sensitivities of the 6 studies for the analysis of IP-10 as a biomarker of TB. Statistical method: inverse variance of the random effects model. CI, confidence interval; TB, tuberculosis; IP-10, IFN- $\gamma$-inducible protein 10.

greater the clinical correlation between IP-10 and TB. The $\mathrm{I}^{2}$ was $90.6 \%$, so there is obvious heterogeneity and the random effects model was used for combined analysis. The analysis results showed that the aggregated PLR of the clinical correlation between IP-10 and TB was 4.53 (95\% CI, 1.92-10.70), indicating that the clinical relevance between IP-10 and TB was not high, as shown in Figure 6.

The NLR is the probability of incorrectly diagnosing a negative disease, as the multiple of the negative probability of correctly diagnosing the disease, that is, the ratio of the ratio of false negative rate and true negative rate of the screening results. Therefore, in this study, the smaller the NLR, the higher the correlation between IP-10 and TB. The $\mathrm{I}^{2}$ was $81.8 \%$, there is obvious heterogeneity, and the NLR determined by the random effects model was 0.30 (95\% CI, 0.17-0.52), indicating that IP-10 is more accurate 


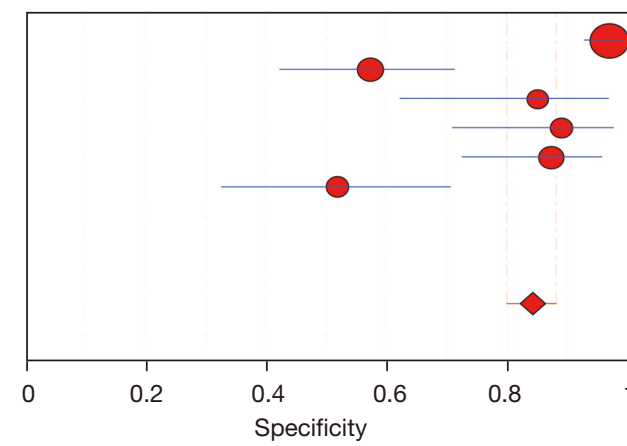

Blauenfeldt

dt $\quad 0.97-(0.93-0.99)$

$\begin{array}{lll}\text { Luo } & 0.57 & (0.42-0.71)\end{array}$

$\begin{array}{lll}\text { La manna } & 0.85 & (0.62-0.97)\end{array}$

$\begin{array}{lll}\text { Balcells } & 0.89 & (0.71-0.98)\end{array}$

$\begin{array}{lll}\text { Nonghanphithak } & 0.87 & (0.73-0.96)\end{array}$

$\begin{array}{lll}\text { Mamishi } & 0.52 & (0.33-0.71)\end{array}$

Pooled specificity $=0.84$ ( 0.80 to 0.88$)$

Chi-square $=64.90 ; \mathrm{df}=5(\mathrm{P}=0.0000)$

Inconsistency (l-square) $=92.3 \%$

Figure 4 Forest plot of the specificities of the 6 studies for the analysis of IP-10 as a biomarker of TB. Statistical method: inverse variance of the random effects model. CI, confidence interval; TB, tuberculosis; IP-10, IFN- $\gamma$-inducible protein 10.

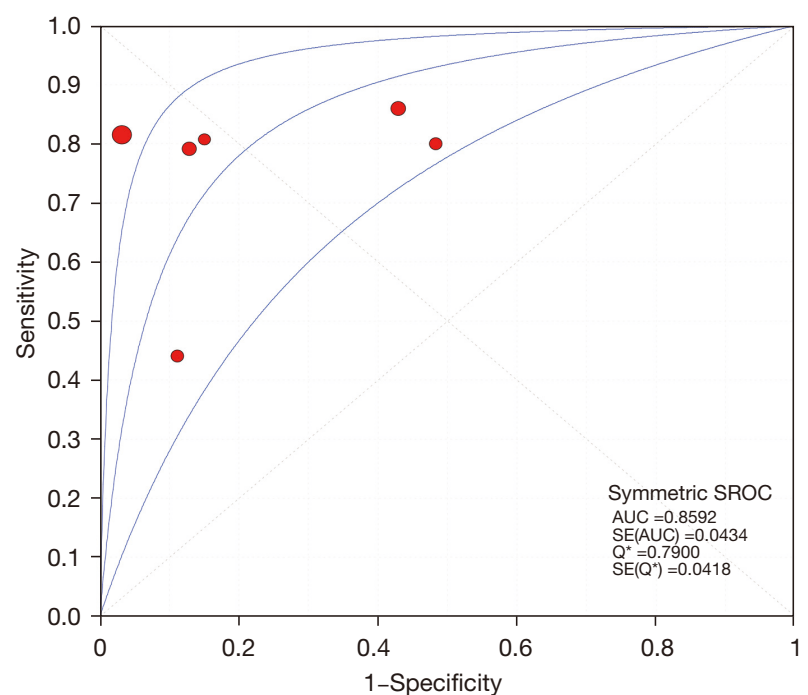

Figure 5 SROC curve for individual studies on the clinical relevance of IP-10 for tuberculosis. SROC, summary receiver operating characteristic; AUC, area under the curve; SE, standard error; IP-10, IFN- $\gamma$-inducible protein 10.

in the screening of TB, as shown in Figure 7.

The DOR reflects the closeness of the relationship between the diagnostic test results and the corresponding disease. The higher the DOR, the greater the likelihood of the disease being diagnosed through screening tests. The $\mathrm{I}^{2}$ was $79.5 \%$, there is obvious heterogeneity, and the random effects model showed that the combined DOR of IP-10 for TB diagnosis was 16.35 (95\% CI, 5.56-48.11), as shown in Figure 8.

\section{Overall analyses of IL-2}

The results of the meta-analysis showed that the sensitivity $\mathrm{I}^{2}$ of the clinical correlation between IL-2 and TB was $66.2 \%$, so the random effects model was used for the combined analysis. The pooled sensitivity was 0.82 (95\% CI, 0.74-0.89) (Figure 9). The specificity $\mathrm{I}^{2}$ was $0.0 \%$, and the fixed effects model was used for binding analysis. The pooled specificity was 0.95 (95\% CI, 0.88-0.98) (Figure 10). The SROC curve showed higher accuracy (SAUC $=0.9666$ ) (Figure 11). The closer the AUC of the SROC curve is to 1.0, the stronger the clinical correlation between IL-2 and TB.

The PLR is the multiple of the correctly diagnosed disease and the incorrectly diagnosed disease in the diagnostic auxiliary examination. Therefore, in this study, the greater the PLR, the greater the clinical correlation between IL-2 and TB. The $\mathrm{I}^{2}$ was $0.0 \%$, so the fixed effects model was used for combined analysis. The analysis results showed that the aggregate PLR of the clinical correlation between IL-2 and TB was 12.63 (95\% CI, 5.78-27.64), indicating that the clinical relevance of IL-2 for TB was higher, as shown in Figure 12.

The NLR is the multiple of the probability of incorrectly diagnosing a negative disease, as the multiple of the negative probability of correctly diagnosing the disease. Therefore, in this study, the smaller the NLR, the higher the correlation between IL-2 and TB. The $\mathrm{I}^{2}$ was $38.3 \%$, and the NLR determined by the fixed effects model was 0.20 (95\% CI, 0.14-0.30), indicating that IL-2 is more accurate in the screening of TB, as shown in Figure 13.

The DOR reflects the closeness of the relationship between the diagnostic test results and the corresponding disease. The higher the DOR, the greater the likelihood of the disease being diagnosed through screening tests. The $\mathrm{I}^{2}$ was $0.0 \%$, and the fixed effects model showed that the combined DOR of IL-2 for TB diagnosis was 67.71 (95\% CI, 25.08-182.79), as shown in Figure 14. 


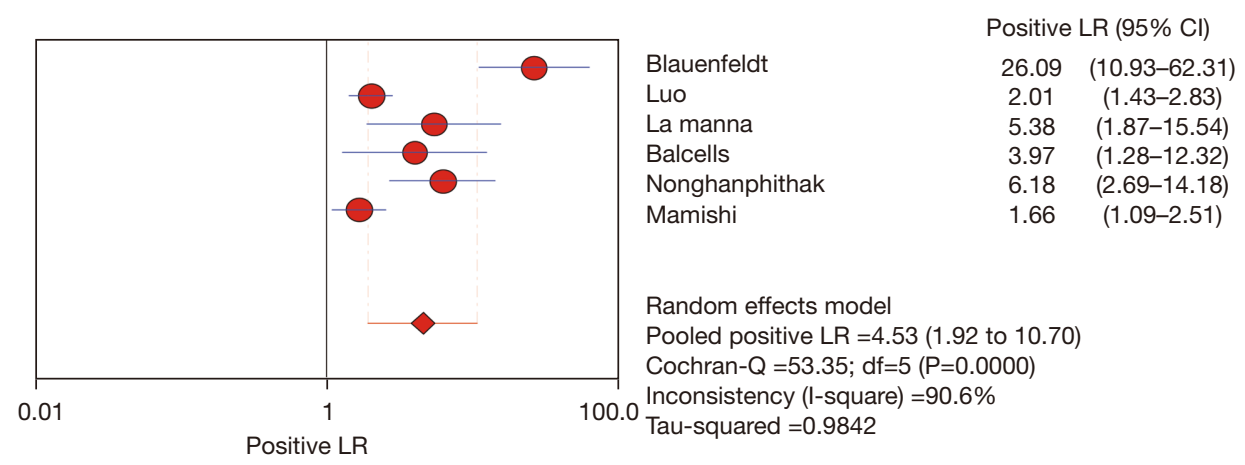

Figure 6 Forest plot of the positive LR for the clinical correlation between IP-10 and tuberculosis. Statistical method: inverse variance of the random effects model (LR and 95\% CI). LR, likelihood ratio; IP-10, IFN- $\gamma$-inducible protein 10; CI, confidence interval.

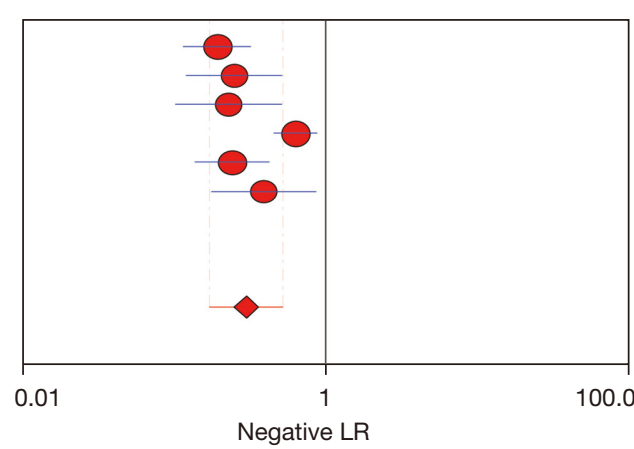

$\begin{array}{lr} & \text { Negativ } \\ \text { Blauenfeldt } & 0.19 \\ \text { Luo } & 0.25 \\ \text { La manna } & 0.23 \\ \text { Balcells } & 0.63 \\ \text { Nonghanphithak } & 0.24 \\ \text { Mamishi } & 0.39 \\ & \\ & \\ \text { Random effects model } & \\ \text { Pooled negative } \mathrm{LR}=0.30(0.17 \text { to } 0.52) \\ \text { Cochran- } \mathrm{Q}=27.43 ; \mathrm{df}=5(\mathrm{P}=0.0000) \\ \text { Inconsistency }(\mathrm{I}-\mathrm{square})=81.8 \% \\ \text { Tau-squared }=0.3732\end{array}$

Figure 7 Forest plot of the negative LR for the clinical correlation between IP-10 and tuberculosis. Statistical method: inverse variance of the random effects model (LR and 95\% CI). LR, likelihood ratio; IP-10, IFN- $\gamma$-inducible protein 10; CI, confidence interval.

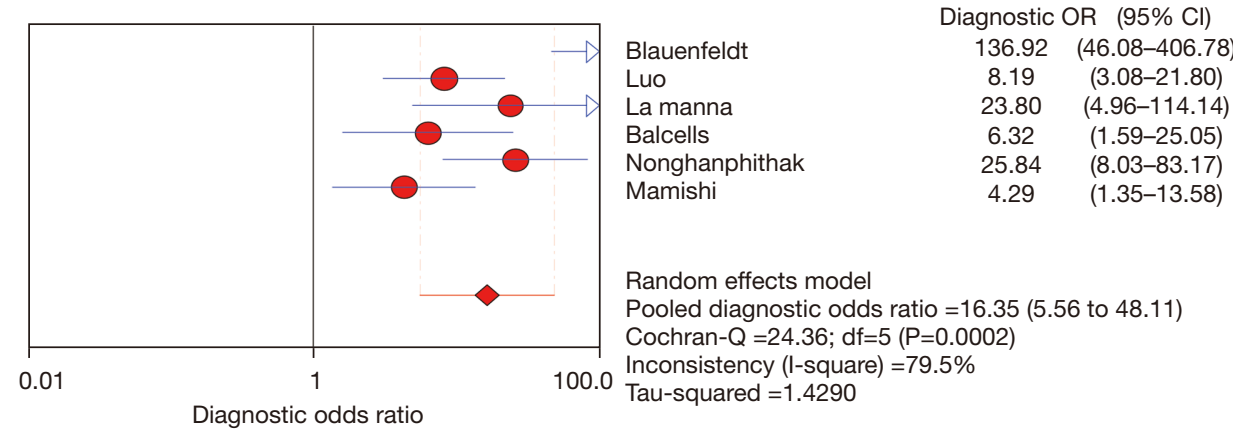

Figure 8 Forest plot of the diagnostic odds ratio for IP-10 in the diagnosis of tuberculosis. Statistical method: inverse variance of the random effects model (diagnostic odds ratio and 95\% CI). IP-10, IFN- $\gamma$-inducible protein 10; CI, confidence interval.

\section{Risk of bias}

The quality assessment found that in terms of patient selection bias, 8 articles were low risk (26-28,30-34) and 1 article had unclear risk (29). In terms of research index test bias, 4 articles were low risk $(26,29,30,34)$ and 5 articles had unclear risk (27,28,31-33). All articles on reference standard bias were low risk. In terms of flow and time bias, 8 articles were low risk (26-28,30-34) and 1 article had unclear risk (29). Regarding the risk of applicability bias, patient selection bias, and reference standard bias, all articles were low risk. Index test bias was low risk in 8 articles (26,28-34), while the risk in 1 article was unclear (27), as shown in Figure 15. 


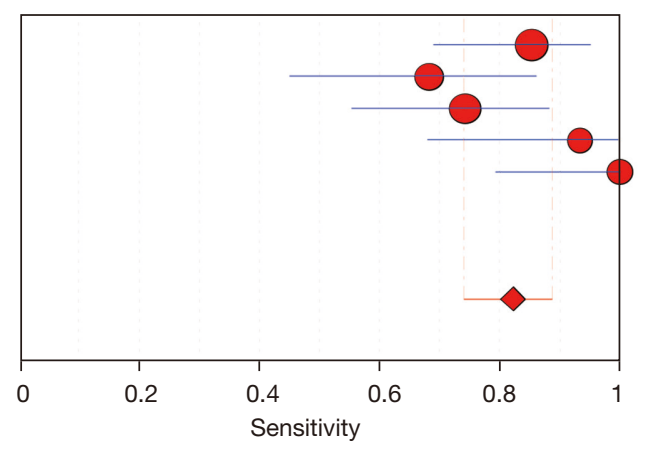

\begin{tabular}{lcc} 
& \multicolumn{2}{c}{ Sensitivity $(95 \% \mathrm{Cl})$} \\
Wang & 0.85 & $(0.69-0.95)$ \\
Balcells & 0.68 & $(0.45-0.86)$ \\
La Manna & 0.74 & $(0.55-0.88)$ \\
Nausch & 0.93 & $(0.68-1.00)$ \\
Kamakia & 1.00 & $(0.79-1.00)$
\end{tabular}

Pooled sensitivity $=0.82$ ( 0.74 to 0.89 )

Chi-square $=11.85 ; \mathrm{df}=4(\mathrm{P}=0.0185)$

Inconsistency (I-square) $=66.2 \%$

Figure 9 Forest plot of the sensitivities of the 5 studies for the analysis of IL-2 as a biomarker of TB. Statistical method: inverse variance of the random effects model. IL-2, interleukin 2; TB, tuberculosis; CI, confidence interval.

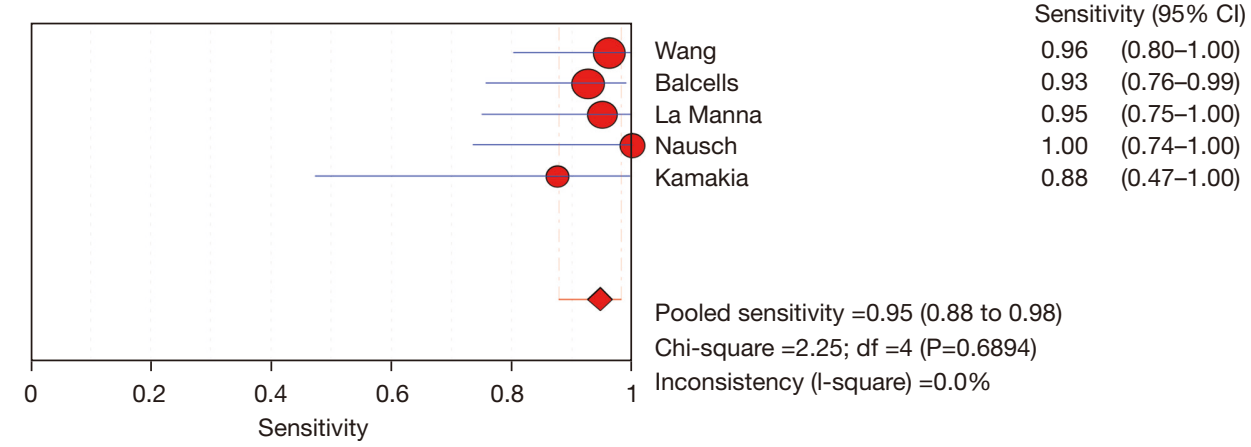

Figure 10 Forest plot of the specificities of the 5 studies for the analysis of IL-2 as a biomarker of TB. Statistical method: inverse variance of the random effects model. IL-2, interleukin 2; TB, tuberculosis; CI, confidence interval.

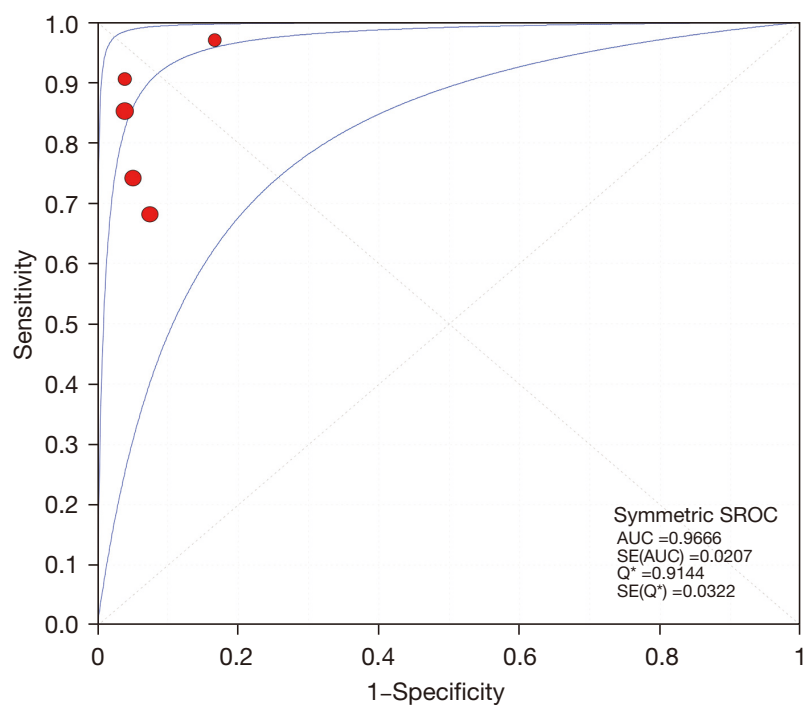

Figure 11 SROC curve for individual studies on the clinical relevance of IL-2 for tuberculosis. SROC, summary receiver operating characteristic; AUC, area under the curve; SE, standard error; IL-2, interleukin 2 .

\section{Discussion}

In recent years, there has been a trend of recurrence of TB outbreaks. The prevalence of drug-resistant TB and the emergence of co-infection with TB have made this trend more serious. Therefore, there is an urgent need for effective and rapid diagnostic methods to detect TB infection and further distinguish active disease and LTBI. There is also a need for effective standard antiTB chemotherapies for active TB patients and preventive treatment for LTBI. In this study, we investigated the application of TB-related markers IP-10 and IL-2 for the diagnosis of TB. We also preliminarily speculated that the combined application of the two may be used to distinguish active TB, LTBI, and healthy people.

Accurate diagnosis methods for TB patients and accurate prediction tests determining which LTBI patients will develop TB will ensure that preventive treatment can target those who are at increased risk of $\mathrm{TB}$, while saving those with the lowest risk from costs, burden, and the impact of 




$\begin{array}{lcc} & \text { Positive LR }(95 \% \mathrm{Cl}) \\ \text { Wang } & 22.18 & (3.23-152.32) \\ \text { Balcells } & 9.20 & (2.35-36.00) \\ \text { La Manna } & 14.84 & (2.17-1.1 .38) \\ \text { Nausch } & 23.56 & (1.55-358.60) \\ \text { Kamakia } & 5.82 & (1.35-25.16) \\ & & \\ \text { Fixed effects model } & \\ \text { Pooled positive LR }=12.63(5.78 \text { to } 27.64) \\ \text { Cochran-Q =1.84; df=4 }(\mathrm{P}=0.7653) \\ \text { Inconsistency (I-square) }=0.0 \% \\ \end{array}$

Figure 12 Forest plot of the positive LR for the clinical correlation between IL-2 and tuberculosis. Statistical method: inverse variance of the random effects model (LR and 95\% CI). LR, likelihood ratio; IL-2, interleukin 2; CI, confidence interval.

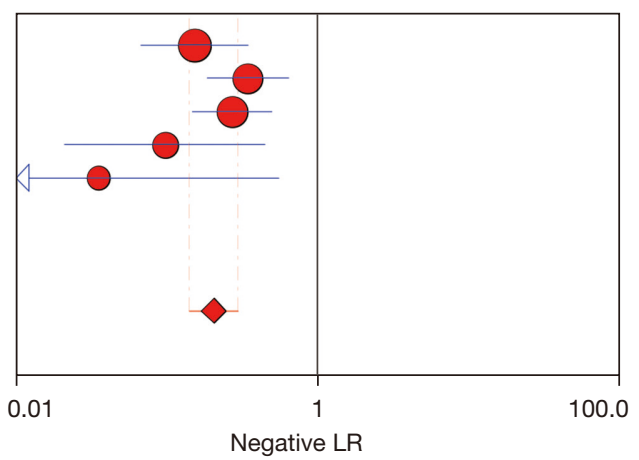

\begin{tabular}{lll} 
& \multicolumn{2}{c}{ Negative LR $(95 \% \mathrm{Cl})$} \\
Wang & 0.15 & $(0.07-0.34)$ \\
Balcells & 0.34 & $(0.18-0.64)$ \\
La Manna & 0.27 & $(0.15-0.50)$ \\
Nausch & 0.10 & $(0.02-0.45)$ \\
Kamakia & 0.04 & $(0.00-0.55)$ \\
& \\
& \\
Fixed effects model & \\
Pooled negative $\mathrm{LR}=0.20(0.14$ to 0.30$)$ \\
Cochran- $\mathrm{Q}=6.49 ; \mathrm{df}=4(\mathrm{P}=0.1656)$ \\
Inconsistency $(\mathrm{I}-\mathrm{square})=38.3 \%$ \\
\end{tabular}

Figure 13 Forest plot of the negative LR for the clinical correlation between IL-2 and tuberculosis. Statistical method: inverse variance of the random effects model (LR and 95\% CI). LR, likelihood ratio; IL-2, interleukin 2; CI, confidence interval.

side effects from unnecessary intervention. Current tests for TB infection, including IGRAs and TSTs, have poor predictive ability due to their low specificity for new TB. Furthermore, they are not cost-effective for treating the approximately 2 billion people potentially infected with TB worldwide, so preventive treatment with more specific biomarkers may be a more feasible alternative $(24,35)$. Predictive testing can be used for large-scale TB screening activities or contact investigations at the community level, but it may also be useful for symptomatic individuals who have been investigated but have not been found to have active $\mathrm{TB}$ at the time of testing.

In this study, it was found that the inflammatory factors IP-10 and IL-2 have high diagnostic value for TB. Although the amount secreted after being stimulated by TB antigens cannot distinguish between active TB and LTBI, they have a higher positive rate of diagnosis, and the combination of IP10 and IL-2 can play a complementary role. The diagnostic value of IP-10 and IL-2 is consistent with the results of other studies, and related studies have found a significant increase in TB pleurisy patients and positive responders $(36,37)$. IP-10 alone can differentiate and diagnose TB patients and healthy people, while IL-2 has a good differential diagnosis ability for healthy people and LTBI patients, they may be good biomarkers for tuberculosis. At the same time, it can be seen from the analysis results that the sensitivity, specificity, and AUCs were different, and the diagnostic ability of the 2 markers in the same population should be further analyzed in the future.

Our systematic review has some limitations. Publication bias was not evaluated, and existing methods such as funnel plots or regression tests are not helpful for diagnostic accuracy studies. In addition, few biomarkers (only IP-10 and IL-2) were included in the study, and the number of articles included in the study was small, it suggests that IP10 and IL-2 are still lacking in popularization and application. In the future, large-sample, multi-center controlled studies should be conducted to more comprehensively evaluate the 


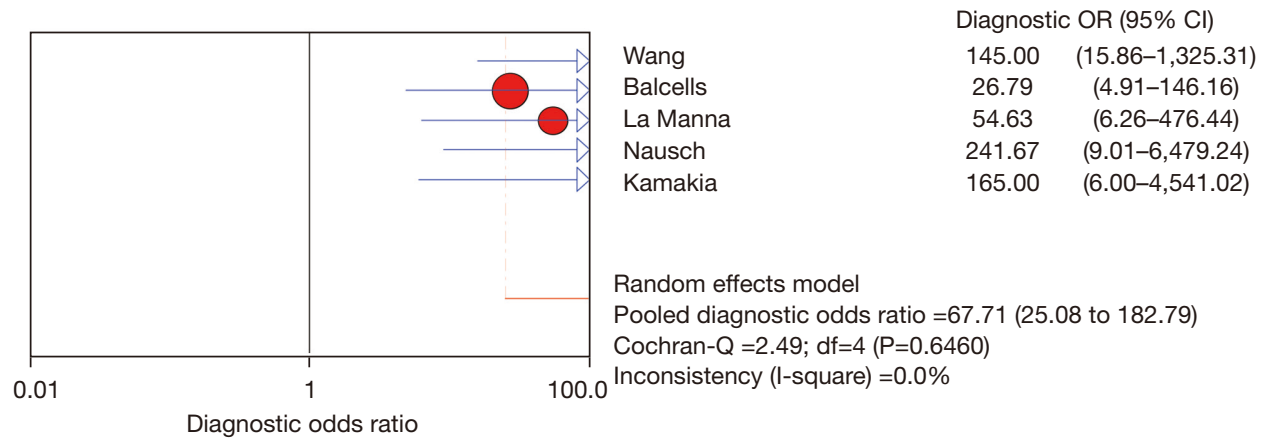

Figure 14 Forest plot of the diagnostic odds ratio for IL-2 in the diagnosis of tuberculosis. Statistical method: inverse variance of the random effects model (diagnostic odds ratio and 95\% CI). IL-2, interleukin 2; CI, confidence interval.

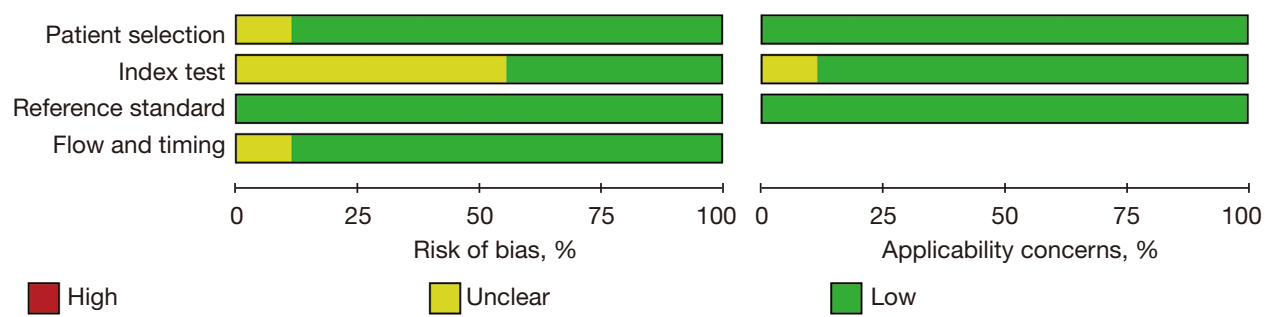

Figure 15 The intensity and distribution of the quality and risk of bias of the articles included in the study.

clinical relevance of TB biomarkers.

\section{Conclusions}

In summary, the results of this systematic review showed that although IP-10 and IL-2 as biomarkers have good clinical relevance to $\mathrm{TB}$, more attention needs to be paid to improving the research design and quality of TB biomarker studies. In addition, such studies should be multi-center studies conducted in different geographic locations and populations, so that diagnostic or classification tests can be applied in multiple settings worldwide. Another method is to use a prospective cohort to conduct parallel diagnostic accuracy studies to compare the performance of different classifications or diagnostic tests. This will improve the reliability of the results and the potential for translating research results into clinical practice.

\section{Acknowledgments}

Funding: The work was supported financially by grants from the Foundation of Nantong sanitation and health committee (QA2020038, MB2019029).

\section{Footnote}

Reporting Checklist: The authors have completed the PRISMA-DTA reporting checklist. Available at https://apm. amegroups.com/article/view/10.21037/apm-21-3739/rc

Conflicts of Interest: All authors have completed the ICMJE uniform disclosure form (available at https://apm. amegroups.com/article/view/10.21037/apm-21-3739/coif). The authors have no conflicts of interest to declare.

Ethical Statement: The authors are accountable for all aspects of the work in ensuring that questions related to the accuracy or integrity of any part of the work are appropriately investigated and resolved.

Open Access Statement: This is an Open Access article distributed in accordance with the Creative Commons Attribution-NonCommercial-NoDerivs 4.0 International License (CC BY-NC-ND 4.0), which permits the noncommercial replication and distribution of the article with the strict proviso that no changes or edits are made and the original work is properly cited (including links to both the 
formal publication through the relevant DOI and the license). See: https://creativecommons.org/licenses/by-nc-nd/4.0/.

\section{References}

1. Glaziou P, Floyd K, Raviglione MC. Global Epidemiology of Tuberculosis. Semin Respir Crit Care Med 2018;39:271-85.

2. Lönnroth K, Raviglione M. The WHO's new End TB Strategy in the post-2015 era of the Sustainable Development Goals. Trans R Soc Trop Med Hyg 2016;110:148-50.

3. Denkinger CM, Schumacher SG, Gilpin C, et al. Guidance for the Evaluation of Tuberculosis Diagnostics That Meet the World Health Organization (WHO) Target Product Profiles: An Introduction to WHO Process and Study Design Principles. J Infect Dis 2019;220:S91-8.

4. Havelaar AH, Kirk MD, Torgerson PR, et al. World Health Organization Global Estimates and Regional Comparisons of the Burden of Foodborne Disease in 2010. PLoS Med 2015;12:e1001923.

5. World Health Organization. WHO guidelines on tuberculosis infection prevention and control 2019 update. Geneva: License 2019; CC BY-NC-SA 3.0 IGO.

6. Ho PL, Sin WC, Chan JF, et al. Severe influenza A H7N9 pneumonia with rapid virological response to intravenous zanamivir. Eur Respir J 2014;44:535-7.

7. John CA. Realizing the World Health Organization's End TB Strategy (2016-2035): How Can Social Approaches to Tuberculosis Elimination Contribute to Progress in Asia and the Pacific? Trop Med Infect Dis 2019;4:28.

8. Al Abri S, Kasaeva T, Migliori GB, et al. Tools to implement the World Health Organization End TB Strategy: Addressing common challenges in high and low endemic countries. Int J Infect Dis 2020;92S:S60-8.

9. Frahm M, Goswami ND, Owzar K, et al. Discriminating between latent and active tuberculosis with multiple biomarker responses. Tuberculosis (Edinb) 2011;91:250-6.

10. Mahmoudi S, Pourakbari B, Mamishi S. Interferon Gamma Release Assay in response to PE35/PPE68 proteins: a promising diagnostic method for diagnosis of latent tuberculosis. Eur Cytokine Netw 2017;28:36-40.

11. Wergeland I, Pullar N, Assmus J, et al. IP-10 differentiates between active and latent tuberculosis irrespective of HIV status and declines during therapy. J Infect 2015;70:381-91.

12. Mamishi S, Pourakbari B, Teymuri M, et al. Diagnostic accuracy of IL-2 for the diagnosis of latent tuberculosis: a systematic review and meta-analysis. Eur J Clin Microbiol Infect Dis 2014;33:2111-9.

13. Won EJ, Choi JH, Cho YN, et al. Biomarkers for discrimination between latent tuberculosis infection and active tuberculosis disease. J Infect 2017;74:281-93.

14. Chen T, Li Z, Yu L, et al. Profiling the human immune response to Mycobacterium tuberculosis by human cytokine array. Tuberculosis (Edinb) 2016;97:108-17.

15. Hur YG, Kang YA, Jang SH, et al. Adjunctive biomarkers for improving diagnosis of tuberculosis and monitoring therapeutic effects. J Infect 2015;70:346-55.

16. Ruhwald M, Dominguez J, Latorre I, et al. A multicentre evaluation of the accuracy and performance of IP10 for the diagnosis of infection with M. tuberculosis. Tuberculosis (Edinb) 2011;91:260-7.

17. Mamishi S, Pourakbari B, Marjani M, et al. Diagnosis of latent tuberculosis infection among immunodeficient individuals: review of concordance between interferongamma release assays and the tuberculin skin test. Br J Biomed Sci 2014;71:115-24.

18. Chegou NN, Heyckendorf J, Walzl G, et al. Beyond the IFN- $\gamma$ horizon: biomarkers for immunodiagnosis of infection with Mycobacterium tuberculosis. Eur Respir J 2014;43:1472-86.

19. Adetifa IM, Lugos MD, Hammond A, et al. Comparison of two interferon gamma release assays in the diagnosis of Mycobacterium tuberculosis infection and disease in The Gambia. BMC Infect Dis 2007;7:122.

20. Whittaker E, Gordon A, Kampmann B. Is IP-10 a better biomarker for active and latent tuberculosis in children than IFNgamma? PLoS One 2008;3:e3901.

21. Chiappini E, Della Bella C, Bonsignori F, et al. Potential role of M. tuberculosis specific IFN- $\gamma$ and IL-2 ELISPOT assays in discriminating children with active or latent tuberculosis. PLoS One 2012;7:e46041.

22. Gourgouillon N, de Lauzanne A, Cottart $\mathrm{CH}$, et al. TNF- $\alpha / \mathrm{IL}-2$ ratio discriminates latent from active tuberculosis in immunocompetent children: a pilot study. Pediatr Res 2012;72:370-4.

23. Hur YG, Gorak-Stolinska P, Ben-Smith A, et al. Combination of cytokine responses indicative of latent TB and active TB in Malawian adults. PLoS One 2013;8:e79742.

24. Tebruegge M, Dutta B, Donath S, et al. MycobacteriaSpecific Cytokine Responses Detect Tuberculosis Infection and Distinguish Latent from Active Tuberculosis. Am J Respir Crit Care Med 2015;192:485-99. 
25. Chegou NN, Detjen AK, Thiart L, et al. Utility of host markers detected in Quantiferon supernatants for the diagnosis of tuberculosis in children in a high-burden setting. PLoS One 2013;8:e64226.

26. Balcells ME, Ruiz-Tagle C, Tiznado C, et al. Diagnostic performance of GM-CSF and IL-2 in response to longterm specific-antigen cell stimulation in patients with active and latent tuberculosis infection. Tuberculosis (Edinb) 2018;112:110-9.

27. Luo J, Zhang M, Yan B, et al. Diagnostic performance of plasma cytokine biosignature combination and MCP-1 as individual biomarkers for differentiating stages Mycobacterium tuberculosis infection. J Infect 2019;78:281-91.

28. Kamakia R, Kiazyk S, Waruk J, et al. Potential biomarkers associated with discrimination between latent and active pulmonary tuberculosis. Int J Tuberc Lung Dis 2017;21:278-85.

29. La Manna MP, Orlando V, Li Donni P, et al. Identification of plasma biomarkers for discrimination between tuberculosis infection/disease and pulmonary non tuberculosis disease. PLoS One 2018;13:e0192664.

30. Blauenfeldt T, Wagner D, Aabye M, et al. Thermostability of IFN- $\gamma$ and IP-10 release assays for latent infection with Mycobacterium tuberculosis: A TBnet study. Tuberculosis (Edinb) 2016;98:7-12.

31. Mamishi S, Mahmoudi S, Banar M, et al. Diagnostic accuracy of interferon (IFN)- $\gamma$ inducible protein 10 (IP-10) as a biomarker for the discrimination of active and latent tuberculosis. Mol Biol Rep 2019;46:6263-9.

32. Nausch N, Lundtoft C, Schulz G, et al. Multiple cytokines for the detection of Mycobacterium tuberculosis infection in children with tuberculosis. Int J Tuberc Lung Dis 2017;21:270-7.

33. Nonghanphithak D, Reechaipichitkul W, Namwat W, et al. Chemokines additional to IFN- $\gamma$ can be used to differentiate among Mycobacterium tuberculosis infection possibilities and provide evidence of an early clearance phenotype. Tuberculosis (Edinb) 2017;105:28-34.

34. Wang S, Li Y, Shen Y, et al. Screening and identification of a six-cytokine biosignature for detecting TB infection and discriminating active from latent TB. J Transl Med 2018;16:206.

35. Sumner T, Bozzani F, Mudzengi D, et al. Estimating the Impact of Tuberculosis Case Detection in Constrained Health Systems: An Example of Case-Finding in South Africa. Am J Epidemiol 2019;188:1155-64.

36. Lighter J, Rigaud M, Huie M, et al. Chemokine IP-10: an adjunct marker for latent tuberculosis infection in children. Int J Tuberc Lung Dis 2009;13:731-6.

37. Alessandri AL, Souza AL, Oliveira SC, et al. Concentrations of CXCL8, CXCL9 and s'TNFR1 in plasma of patients with pulmonary tuberculosis undergoing treatment. Inflamm Res 2006;55:528-33.

(English Language Editor: C. Betlazar-Maseh)
Cite this article as: $\mathrm{Xu} \mathrm{F,} \mathrm{Ni} \mathrm{M,} \mathrm{Qu} \mathrm{S,} \mathrm{Duan} \mathrm{Y,} \mathrm{Zhang} \mathrm{H,}$ Qin Z. Molecular markers of tuberculosis and their clinical relevance: a systematic review and meta-analysis. Ann Palliat Med 2022;11(2):532-543. doi: 10.21037/apm-21-3739 